\title{
Autoantibodies Against ApoB-100 as a New Marker of Coronary Vulnerable Plaque
}

\author{
Genshi Egusa
}

Genshi Egusa Clinic, Hiroshima, Japan

\section{See article vol. 28: 000-000}

According to the 2019 Vital Statistics, of all deaths in Japan, $15.3 \%$ are caused by heart disease and $7.9 \%$ by cerebrovascular disease. Myocardial infarction accounts for $15.2 \%$ of heart disease mortality, whereas cerebral infarction mortality accounts for $55.6 \%$ of cerebrovascular disease mortality ${ }^{1)}$. The events in atherosclerotic diseases, such as myocardial infarction and cerebral infarction are triggered by the rupture of cholesterol-rich plaques, which results in occlusion by thrombus. Thus, the early detection of vulnerable plaques is crucial to prevent such events.

There are two methods for predicting vulnerable plaques in high-risk patients with coronary artery disease. The first one involves the identification of coronary plaque properties via intravascular ultrasound (IVUS). The vulnerable plaque is characterized by the presence of large necrotic lipid cores covered with a thin fibrous cap ${ }^{2}$. These plaques are at high risk of rupture with subsequent thrombus formation caused by cytokine release due to inflammation, mechanical stress to blood vessels, and so forth. Vulnerable plaques are also characterized by a decrease in fibrotic tissue and an increase in lipid components and plaque burden.

The usefulness of integrated backscatter (IB)IVUS, virtual histology-IVUS, iMAP-IVUS, and so on has been reported for the evaluation of plaque properties. In these methods, the prediction of the histological composition of plaques is visualized as a cross-sectional image. The composition is classified as fibrous, lipid, calcified, necrotic, and so forth and is shown as a color image. The vulnerability of a plaque increases with an increase in the percentage of necrotic and lipid tissue in the total plaque, whereas the stability of a plaque increases with an increase in the percentage of fibrous components ${ }^{3)}$.

The second method for evaluating plaque vulnerability is the measurement of relevant serum biomarkers. Inflammation plays a vital role in plaque progression and rupture. Low-density lipoprotein (LDL) particles that infiltrate the arterial intima are chemically modified and converted to oxidized LDL. The oxidized LDL promotes monocyte adhesion to the vascular wall, infiltration, conversion of macrophages to foam cells, and proliferation. Furthermore, oxidized LDL promotes the proliferation of vascular smooth muscle cells and cell migration to the intima ${ }^{4)}$. Oxidized LDL formation has important implications for the development and progression of plaques.

The $T$ cells in human atherosclerotic plaques are involved in the production of autoantibodies for oxidized LDL. The adaptive immune response to oxidized LDL aggravates inflammation and is involved in the growth of atherosclerotic lesions ${ }^{5)}$. However, reportedly, immunization with oxidized LDL suppresses the progression of atherosclerosis ${ }^{6}$. Previous studies have revealed that the p45 and p210 proteins of oxidized LDL apolipoprotein B-100 (apoB-100) are essential LDL epitopes involved in the immune response of patients with cardiovascular disease ${ }^{7)}$. Indeed, patients with high p45 or p210-IgG antibody titers have a low risk of myocardial infarction ${ }^{8}$. Furthermore, p210-IgG antibody titers were reported to inversely correlate with the severity of coronary artery lesions ${ }^{8)}$. Thus, these antibodies are considered to possess an anti-arteriosclerotic effect. In line with this concept, recent animal experiments have reported that the apoB-100 p210 antibody causes plaque stabilization through the increased efflux of cholesterol from macrophages and decreased inflammatory cytokine levels ${ }^{9)}$. The apoB-100 autoantibodies may 
increase plaque stability and contribute to the suppression of atherosclerotic events. However, the association between morphologically evaluated plaque stability and apoB-100 autoantibodies remains to be elucidated.

In this issue of the Journal of Atherosclerosis and Thrombosis, Imai et al. investigated the relationship between plaque morphology evaluated by iMAPIVUS and the serum LDL-apoB autoantibody titer and found that the apoB autoantibody titer could be a useful biomarker of plaque instability ${ }^{10)}$.

In 88 male patients undergoing elective percutaneous coronary intervention (PCI), iMAPIVUS was used to analyze the plaque morphology of the most stenosed segments as determined by coronary angiography. Furthermore, serum anti-ApoB-100 autoantibody concentration against native and malondialdehyde (MDA)-modified peptides (p45 and p210) were measured via enzyme-linked immunosorbent assay. The average composition of the target plaque was $32.8 \%$ necrotic, $11.7 \%$ lipid, $53 \%$ fibrotic, and $3.0 \%$ calcified.

Considering all the subjects, the results indicate that plaque burden had a significant inverse correlation with $\operatorname{IgG}_{\mathrm{N}-\mathrm{p} 45}$, IgGN-p210 and IgGMDA-p45, and IgGMDA-p210 levels. However, no significant correlation was noted with respect to other components.

Given the plaque-stabilizing effect of statins, the subjects were divided into a statin group (that had taken statin for more than a month before PCI) and a non-statin group (that had taken statin for less than a month). In the non-statin group $(n=45), \operatorname{IgGN}_{-\mathrm{p} 45}$ level showed a significant inverse correlation with plaque burden and necrotic components and a significant positive correlation with fibrotic components. IgGN-p210 levels showed a significant inverse correlation with plaque burden and total plaque volume and a positive correlation with fibrotic components. Nevertheless, in the statin group, no significant association was noted between plaque composition and these antibody titers ${ }^{10)}$.

These results suggest that $\operatorname{IgGN}_{\mathrm{N}-\mathrm{p} 45}$ and $\mathrm{IgGN}_{\mathrm{N}-\mathrm{p} 210}$ serum levels can be used as predictors of vulnerable plaques in male patients with a high risk of coronary artery disease without statin treatment. Animal experiments have revealed that immunization with apoB-100 peptides suppresses atherosclerotic lesions ${ }^{11)}$. Based on these results, the suppression of the inflammatory reaction by oxidized LDL at lesion sites is insufficient when LDL-apoB autoantibody titers are low, and this may be related to plaque instability and susceptibility to plaque growth.

These results are interesting, but further investigations are required in terms of the following: (1) The association between LDL-apoB autoantibody and carotid intima-media thickness has been reported $^{12)}$, and ischemic stroke is one of the major causes of deaths among Japanese people ${ }^{1)}$. Based on these facts, can these antibodies also be used as indicators of carotid plaque vulnerability given their usefulness regarding coronary plaque vulnerability? (2) Can these values be predictors of myocardial infarction and ischemic stroke in prospective studies in the Japanese population? (3) As this study only involved male subjects, can these markers also be used as indicators of vulnerable plaques in women as sex differences in serum anti-oxidized LDL antibodies levels have been reported ${ }^{13,14)}$ ?

Only a few studies have examined biomarkers that can serve as indicators of the vulnerable plaque evaluated via IVUS; we expect further studies on this topic.

\section{Conflicts of Interests}

None.

\section{References}

1) Health, Labour and Welfare Statistics Association. National hygiene trends 2019/2020. Journal of health and welfare statistics, 2019; 66(9)

2) Virmani R, Kolodgie FD, Burke AP, Farb A, Schwartz M. Lessons from sudden coronary death. A comprehensive morphological classification scheme for atherosclerotic lesions. Arterioscler Thromb Vasc Biol, 2000; 20: $1262-$ 1275

3) Virmani R, Burke AP, Farb A, Kolodgie FD. Pathology of the vulnerable plaque. J Am Coll Cardiol, 2006; 47: C13C18

4) Gistera A, Hansson GK. The immunology of atherosclerosis. Nature reviews nephrology, 2017; 13: 368-380

5) Stemme S, Faber BF, Holm J, Wiklund O, Witztum JL, Hansson GK. T lymphocytes from human atherosclerotic plaques recognize oxidized low density lipoprotein. Proc Natl Acad Sci, 1995; 92: 3893-3897

6) Zhou X, Caligiuri G, Hamsten A, Lefvert AK, Hansson GK. LDL immunization induces T-cell-dependent antibody formation and protection against atherosclerosis. Arterioscler Thromb Vasc Biol, 2001; 21: 108-114

7) Fredrikson GN, Hedblad B, Berglund G, Alm R, Ares M, Cercek B, Chyu KY, Shah PK, Nilsson J. Identification of immune responses against aldehyde-modified peptide sequences in apoB associated with cardiovascular disease. Arterioscler Thromb Vasc Biol, 2003; 23: 872-878

8) Sjogren P, Fredrikson GN, Samnegard A, Ericsson CG, Ohrvik J, Fisher RM, Nilsson J, Hamsten A. High plasma concentrations of autoantibodies against native peptide 210 of apoB-100 are related to less coronary atherosclerosis 
and low risk of myocardial infarction. Eur. Heart J, 2008; 29: 2218-2226

9) Zeng Z, Cao B, Guo X, Li W, Li S, Chen J, Zhou W, Zheng C, Wei Y. Apolipoprotein B-100 peptide 210 antibody inhibits atherosclerosis by regulation of macrophages that phagocytized oxidized lipid. Am J Transl Res, 2018; 10: 1817-1828

10) Imai $M$, Kawamura $M$, Kochi I, Matsuoka T, Kihara $S$, Yamamoto H. Anti-ApoB-100 autoantibody is a marker of unstable coronary plaque. J Atheroscler Thromb, 2020; in press. DOI: http://doi.org/10.555/jat.587841

11) Fredrikson GN, Soderberg I, Lindholm M, Dimayuga P, Chyu KY, Shah PK, Nilsson J. Inhibition of atherosclerosis in apoE-null mice by immunization with apoB-100 peptide sequences. Arterioscler Thromb Vasc Biol, 2003; 23: 879-884
12) Fredrikson GN, Hedblad Bo, Berglund G, Alm R, Nilsson JK, Schiopu A, Shah PK, Nilsson J. Association between IgM against an aldehyde-modified peptide in apolipoprotein B-100 and progression of carotid disease. Stroke, 2007; 38: 1495-1500

13) Tinahones FJ, Gomez-Zumaquero JM, Garrido-Sanchez L, Garcia-Fuentes E, Rojo-Martinez G, Esteva I, Ruiz de Adana MS, Cardona F, Soriguer F. Influence of age and sex on levels of anti-oxidized LDL antibodies and antiLDL immune complexes in the general population. J. Lipid Res, 2005; 46: 452-457

14) Su J, Georgiades A, Wu R, Thulin T, Faire U, Frostegard J. Antibodies of IgM subclass to phosphorylcholine and oxidized LDL are protective factors for atherosclerosis in patients with hypertension. Atherosclerosis, 2006; 188: $160-166$ 